\title{
Metodologia de Cálculo do Fator de Forma para Geometrias Tridimensionais Considerando os Efeitos de Obstrução
}

\author{
Fábio Alfaia da Cunha, Pedro Paulo Dunice Van Els e Daniel Back da Trindade \\ Universidade de Brasília, Brasília-DF, Brasil.
}

RESUMO: Em muitas aplicações de engenharia é necessário o conhecimento da transferência de calor por radiação entre superfícies. 0 parâmetro que contabiliza a fração de energia radiativa recebida por um corpo proveniente de outro é chamado fator de forma. Os estudos que envolvem o cálculo do fator de forma se tornam complicados quando são analisadas geometrias complexas, dificultando a obtenção deste parâmetro por meio de soluções analíticas. Para tanto é necessária à utilização de um método de solução numérico. Por conta do exposto, o presente trabalho apresenta uma metodologia numérica que utiliza malhas tetraédricas para o cálculo do fator de forma para geometrias tridimensionais, considerando os efeitos de obstrução. Como será visto, os resultados obtidos com a metodologia proposta são satisfatórios, pois se aproximam de resultados analíticos disponibilizados na literatura.

Palavras-chave: Fator de forma, obstrução; transferência de calor; radiação, simulação numérica.

ABSTRACT: In many engineering applications it is necessary to specify the heat transfer by radiation between different surfaces. The parameter used to measure the radiation energy fraction received by a body is called view factor. The calculations to determine the view factor are tricky whenever complex geometries are accessed, eventually it is not feasible to determine such parameter in an analytic way. Therefore, it is necessary to use a numeric approach. Due to the above, the present work presents a numerical methodology that uses tetrahedral meshes for the calculation of the view factor for threedimensional geometries, which considering obstruction effects. The acquired results with such methodology have proven itself to be suitable, because they tend to the analytical results in the literature.

Keywords: View factor, obstruction, heat transfer, radiation, numerical simulation. 


\section{INTRODUÇÃO}

Situações envolvendo troca de calor por radiação, difusão e convecção são frequentemente encontradas nas engenharias que utilizam máquinas térmicas. 0 dimensionamento ótimo de máquinas térmicas tem sido uma meta perseguida desde os primórdios da engenharia, porém, para que tal dimensionamento ocorra modelos matemáticos complexos devem ser resolvidos, o que demanda técnicas de solução sofisticadas. Quando a transferência de calor por radiação é o mecanismo dominante, um parâmetro chamado fator de forma se torna importante, pois é este que contabiliza a fração de energia radiativa recebida por um corpo proveniente de outro. Sua determinação requer cálculos complexos quando se deseja exatidão para geometrias normalmente encontradas em máquinas industriais e (JOY, 2014).

Muitos trabalhos têm focado no desenvolvimento de métodos computacionais de cálculo do fator. Em Miyanaga e Nakano (2003), por exemplo, é proposto um método de rastreamento de raios para o cálculo do fator de forma especular em ambientes tridimensionais. Segundo os autores o método é preciso para tempo computacional prático para espaços fechados que contenham obstáculos onde ocorrem reflexões especulares complexas. Já em Yang et al. (2014) é estudado a troca de calor entre varetas de combustível nuclear utilizando fluidodinâmica computacional. As varetas de combustível são responsáveis por envolver as pastilhas de urânio, utilizadas dentro dos reatores nucleares para geração de energia nuclear. Neste cenário, o parâmetro fator de forma é importante para os estudos relacionados a energia nuclear, devido a necessidade de controle das taxas de transferência de calor durante o processo.

De fato, o cálculo do fator de forma se torna complicado quando são analisadas geometrias tridimensionais com obstáculos, o que torna a utilização de um método de solução numérico para obtenção do fator de forma quase obrigatória. Assim, este trabalho visa contribuir exatamente neste sentido, propondo uma metodologia numérica de cálculo do fator de forma para geometrias tridimensionais, que considera efeitos de obstrução e utiliza malhas computacionais tetraédricas, que são bastante utilizadas em simulações de fluidodinâmica computacional.

\section{METODOLOGIA NUMÉRICA DE CÁLCULO DO FATOR DE FORMA}

A metodologia de cálculo do fator de forma apresentada a seguir é uma extensão para o caso tridimensional do que foi apresentado em Araújo e Da Cunha (2016). 


\subsection{Equação integral do fator de forma}

De modo geral a troca de calor por radiação entre corpos depende de sua orientação e ainda das suas propriedades de radiação e temperatura. 0 parâmetro que leva em conta a orientação dos corpos é o fator de forma, que possibilita quantificar a fração de energia que sai de uma superfície e atinge uma segunda superfície. O Fator de forma é definido como a fração de radiação que sai da superfície isotérmica, opaca, e difusa 1 (por emissão ou reflexão) e incide diretamente sobre a superfície 2 (absorvida ou refletida) e depende apenas de fatores geométricos (HOWELL e MENGÜÇ, 2011). A expressão geral do fator de forma entre duas superfícies difusas de área infinitesimal está presente na Eq. (1). Os elementos geométricos de apoio a Eq. (1) estão dispostos na Figura 1.

$$
F_{12}=F_{A 1 \rightarrow A 2}=\frac{1}{A_{1}} \int_{A 1} \int_{A 2} \frac{\cos \theta_{1} \cos \theta_{2}}{\pi S^{2}} d A_{1} d A_{2}
$$

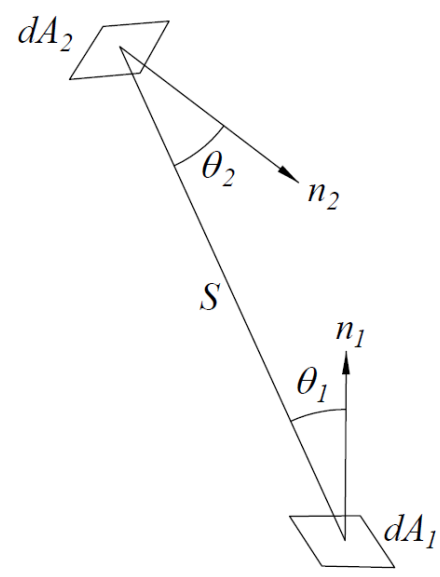

Figura 1. Esquema para cálculo do fator de forma entre superfícies.

\subsection{Equação vetorial aproximada do fator de forma}

Com base na malha computacional apresentada na Figura 2, as integrais da Equação (1) podem ser aproximadas por somatórias da seguinte forma:

$$
F_{i j}=-\frac{1}{\pi A_{i}} \sum_{k=1}^{n 1} \sum_{l=1}^{n 2} \frac{\left(\vec{r}_{k l} \cdot \vec{n}_{u k}\right)\left(\vec{r}_{k l} \cdot \vec{n}_{u l}\right)}{\left(\vec{r}_{k l} \cdot \vec{r}_{k l}\right)^{2}} b_{k l} \Delta A_{k} \Delta A_{l}
$$

Na qual $\vec{r}_{k l}$ é o vetor que conecta os centros dos elementos de área $\Delta A_{k}$ e $\Delta A_{l}$. Já $\vec{n}_{u k}$ e $\vec{n}_{u l}$ são vetores normais unitários a $\Delta A_{k}$ e $\Delta A_{l}$, respectivamente. 0 fator $b_{k l}$ contabiliza a obstrução de radiação entre duas superfícies, de forma que toma valor zero caso exista obstrução e unitário caso contrário. As superfícies "i” e "j” são divididas em n1 e n2 elementos, respectivamente. 


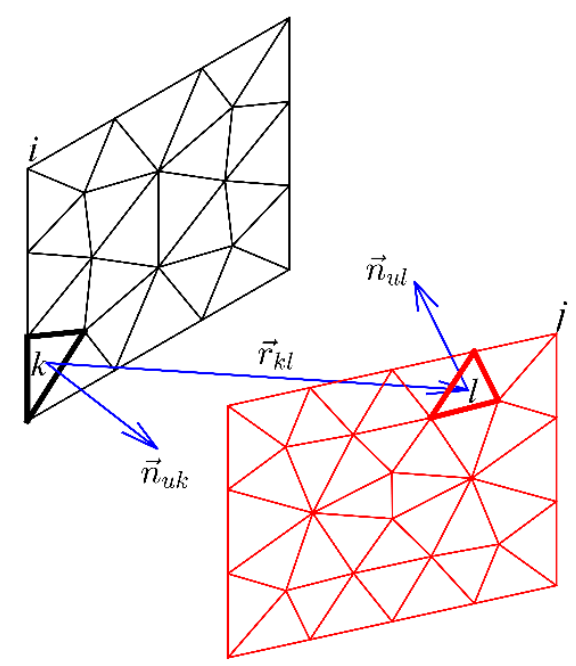

Figura 2. Esquema para o cálculo numérico do fator de forma.

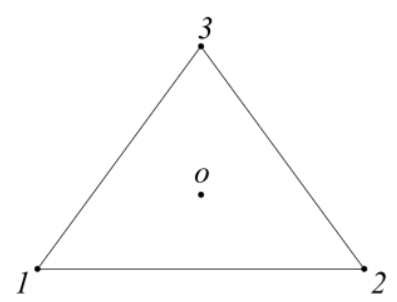

Figura 3. Numeração de um elemento triangular típico " $l$ " ou " $k$ ".

Com base na Figura 3 o vetor normal ao elemento "l" pode ser calculado como: $\vec{n}_{l}=\vec{r}_{o 2, l} \times \vec{r}_{o 3, l}$, em que:

$\vec{r}_{o 2, l}=\left(x_{2, l}-x_{o, l}\right) \mathbf{i}+\left(y_{2, l}-y_{o, l}\right) \mathbf{j}+\left(z_{2, l}-z_{o, l}\right) \mathbf{k}$

$\vec{r}_{o 3, l}=\left(x_{3, l}-x_{o, l}\right) \mathbf{i}+\left(y_{3, l}-y_{o, l}\right) \mathbf{j}+\left(z_{3, l}-z_{o, l}\right) \mathbf{k}$

0 vetor que conecta o baricentro do elemento ao vértice 2 é representado por $\vec{r}_{o 2, l}$ e o vetor que conecta o baricentro do elemento ao vértice 3 é o $\vec{r}_{03, l}$.

Procedendo com o produto vetorial, o vetor normal pode ser expresso em função de suas componentes, $\vec{n}_{l}=n_{l, x} \mathbf{i}+n_{l, y} \mathbf{j}+n_{l, z} \mathbf{k}$ :

$\left[\begin{array}{l}n_{l, x} \\ n_{l, y} \\ n_{l, z}\end{array}\right]=\left[\begin{array}{l}\left(y_{2, l}-y_{0, l}\right)\left(z_{3, l}-z_{0, l}\right)-\left(y_{3, l}-y_{0, l}\right)\left(z_{2, l}-z_{0, l}\right) \\ \left(x_{3, l}-x_{0, l}\right)\left(z_{2, l}-z_{0, l}\right)-\left(x_{2, l}-x_{0, l}\right)\left(z_{3, l}-z_{0, l}\right) \\ \left(x_{2, l}-x_{0, l}\right)\left(y_{3, l}-y_{0, l}\right)-\left(x_{3, l}-x_{0, l}\right)\left(y_{2, l}-y_{0, l}\right)\end{array}\right]$ 
O vetor normal unitário é então obtido: $\vec{n}_{u l}=\vec{n}_{l} /\left|\vec{n}_{l}\right|$.

As áreas dos elementos triangulares podem ser calculadas com base na magnitude dos vetores normais:

$\Delta A_{k}=(3 / 2)\left|\vec{n}_{k}\right|$

$\Delta A_{l}=(3 / 2)\left|\vec{n}_{l}\right|$

As áreas das superfícies " $i$ " e " $j$ " são calculadas pelas somatórias: $A_{i}=\sum_{k=1}^{n 1} \Delta A_{k}$ e $A_{j}=\sum_{l=1}^{n 2} \Delta A_{l}$.

$\mathrm{O}$ vetor $\vec{r}_{k l}$, que conecta os centros dos elementos triangulares " $k$ " e "l" de contorno, é definido com base nas coordenadas centrais de cada elemento de contorno:

$\vec{r}_{k l}=\left(x_{o, l}-x_{o, k}\right) \mathbf{i}+\left(y_{o, l}-y_{o, k}\right) \mathbf{j}+\left(z_{o, l}-z_{o, k}\right) \mathbf{k}$

O centro de cada elemento " $l$ " é obtido como:

$x_{o, l}=\left(x_{1, l}+x_{2, l}+x_{3, l}\right) / 3$

$y_{o, l}=\left(y_{1, l}+y_{2, l}+y_{3, l}\right) / 3$

$z_{o, l}=\left(z_{1, l}+z_{2, l}+z_{3, l}\right) / 3$

Todos os procedimentos desenvolvidos para o elemento "l" podem ser realizados de igual forma para o elemento " $k$ ".

\subsection{Cálculo do fator de Obstrução $\left(b_{k l}\right)$}

0 cálculo do fator de obstrução $b_{k l}$ é realizado em duas etapas. Na primeira etapa são verificados os elementos obstruídos pelo próprio elemento emissor de radiação e na segunda etapa são verificados os elementos obstruídos por outros elementos. Um exemplo prático, com uma geometria e uma malha de elementos considerando as duas etapas é apresentado a seguir.

\subsubsection{Geometria e malha de referência para o cálculo da obstrução.}

Na Figura 4 é apresentada uma geometria de base para descrição da metodologia de cálculo do fator de forma. $\mathrm{O}$ eixo Z está normal ao plano XY, e a espessura da geometria, na direção Z, é 0,5m. A malha de elementos tetraédricos do caso é apresentada na Figura 5. Cada elemento triangular do contorno é uma subparte de um elemento tetraédrico. 


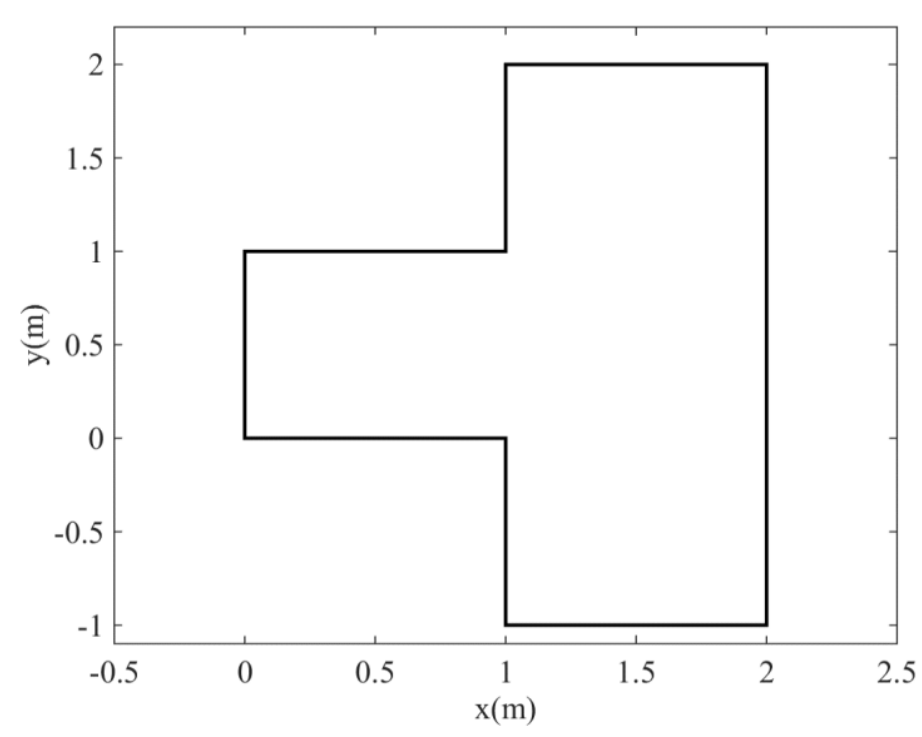

Figura 4. Geometria para descrição da metodologia.

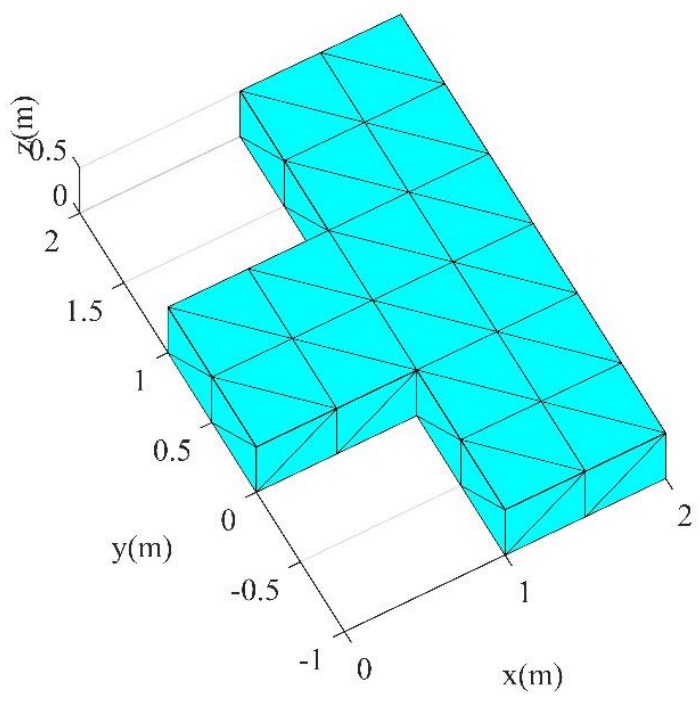

Figura 5. Malha de elementos finitos.

\subsubsection{Determinação dos elementos obstruídos pelo próprio elemento emissor.}

A radiação emitida a partir de um elemento de superfície cobre somente um hemisfério, então, a superfície do elemento emissor de radiação deve obstruir outros elementos. Para a determinação dos elementos de superfície obstruídos pela própria superfície emissora posiciona-se um sistema de coordenadas no centro de um elemento emissor. 0 eixo " $\mathrm{x}$ " do sistema de coordenadas global é alinhado com o vetor normal deste segmento. Na Figura 6 é ilustrado o posicionamento do sistema coordenado referenciado, considerando o elemento triangular 4. Todos os elementos com " $\mathrm{x}_{0} \leq 0$ " estão obstruídos pelo elemento emissor. Na Figura 6 também são apresentados todos os vetores $\vec{r}_{k l}$ que partem do centro do elemento triangular emissor para o centro de todos os elementos triangulares não obstruídos. 


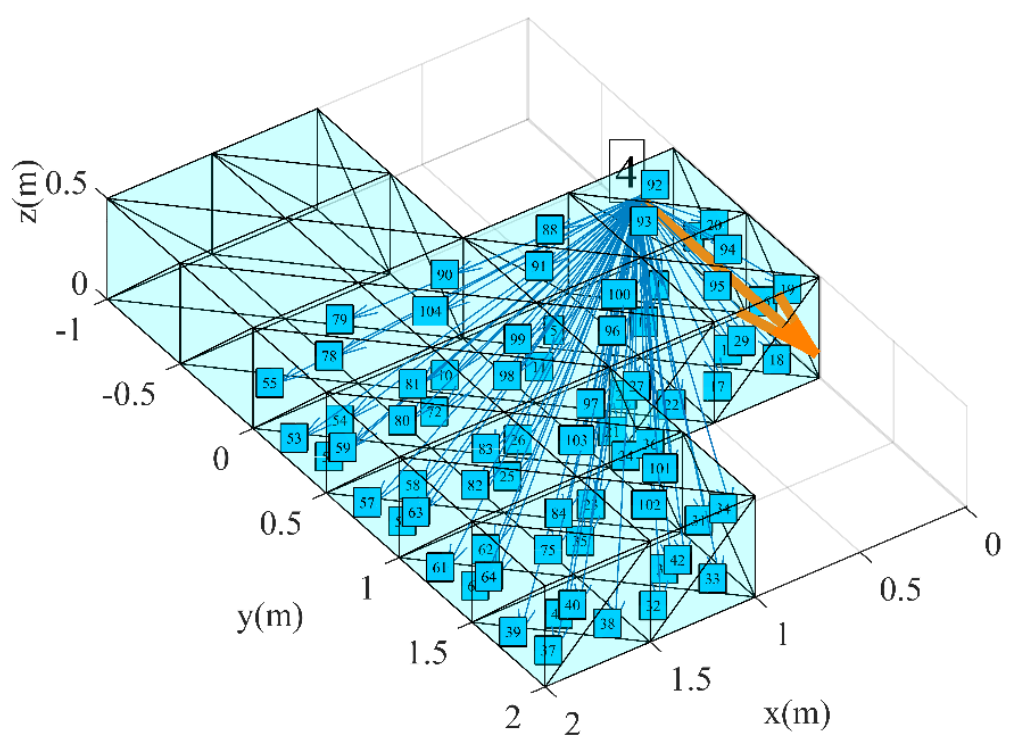

Figura 6. Os elementos rotulados em azul não estão obstruídos pelo elemento emissor.

\subsubsection{Determinação dos elementos obstruídos por outros elementos triangulares.}

Cada vetor $\vec{r}_{k l}$ presente na Figura 6 está associado a um par de elementos triangulares. A verificação de obstrução inicia pelo par de elementos com menor $\left|\vec{r}_{k l}\right|$, que não deve estar obstruído por nenhum outro elemento, e progride para os demais pares em ordem crescente de $\left|\vec{r}_{k l}\right|$.

Uma vez definida a ordem de verificação de pares, para avaliar efetivamente a obstrução criam-se outros três vetores, todos partindo do centro do elemento emissor (elemento 4) em direção aos vértices do elemento obstrutor (elemento 22), conforme Figura 7. Logicamente o par apresentado na figura não seria o primeiro a ser testado, só foi escolhido para exemplificação do modo de determinação de obstrução. No caso considerado, todos os elementos com centro posicionado dentro da região estabelecida pelos três planos são classificados como obstruídos. No caso exemplo, a radiação emitida pelo elemento 4 não chegaria aos elementos de $30,31,32$, 33 e 34, pois estes estariam obstruídos pelo elemento 22.

Depois que todos os elementos disponíveis para obstrução forem verificados, na ordem anteriormente definida, o procedimento é terminado. Na Figura 8 são ilustras todos os elementos que recebem radiação do elemento 4 e também os elementos obstruídos. 


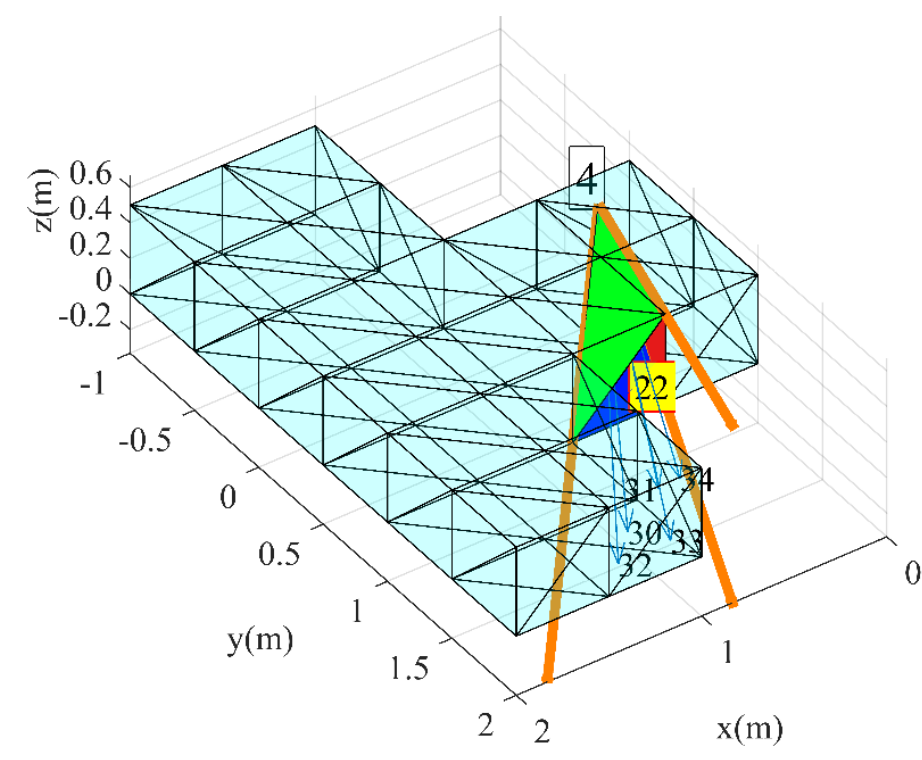

Figura 7. Exemplo de obstrução de radiação.

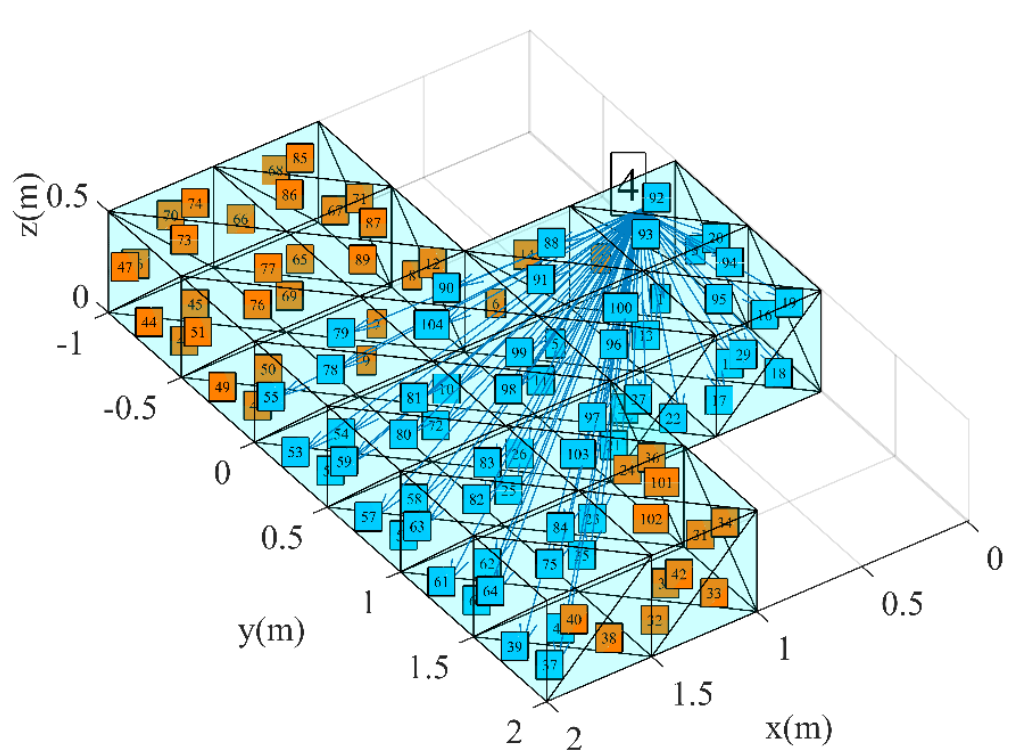

Figura 8. Elementos receptores de radiação estão com rótulo azul e os elementos obstruídos estão com rotulo laranja.

\section{RESULTADOS}

A metodologia de cálculo do fator de forma foi implementada na linguagem do software Matlab para simulação de dois casos de teste. 0 computador utilizado nas simulações possuía processador core i7-6700k, 64 GB de RAM, SSD 256 GB e sistema operacional Windows 10 Pro 64-bit. 


\subsection{Caso de teste 1: Esferas concêntricas.}

O primeiro caso de teste envolve o cálculo do fator de forma entre duas esferas concêntricas, de raios diferentes. 0 interessante neste caso é que a esfera externa emite radiação para si mesma, porém parte da radiação fica obstruída pela esfera interna. Esta ocorrência torna o caso perfeito para teste da metodologia proposta.

A esfera externa será referenciada de 1 (com raio $\mathrm{r}_{1}$ ) e a esfera interna de 2 (com raio $\mathrm{r}_{2}$ ). Para as simulações $r_{2}$ foi mantido igual a 1 e o $r_{1}$ foi variado entre 1,1 e 7 . Duas malhas serão utilizadas nos cálculos, uma malha com elementos de $0,3 \mathrm{~m}$ de tamanho (malha fina) e outra com elementos com 0,45m de tamanho (malha grossa). A malha fina é apresentada na Figura 9, ajustada com transparência para que a superfície interna seja visualizada no seu interior.

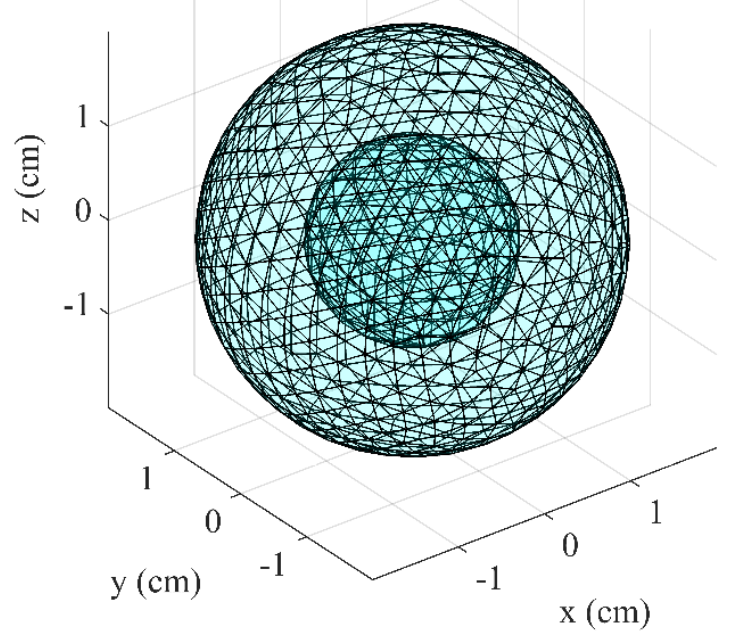

Figura 9. Malha de elementos finitos do caso de esferas concêntricas.

Para este caso o fator de forma $\mathrm{F}_{22}$ é nulo, pois a esfera interna não emite radiação para si mesma. Consequentemente o fator $\mathrm{F}_{21}$ é unitário, por conta da regra da soma. 0 Fator de forma $\mathrm{F}_{11}$ contabiliza a fração de radiação emitida pela superfície externa para si mesma e envolve a obstrução da esfera interna. Pela regra da adição $F_{11}+F_{12}=1$. Pela regra da reciprocidade $\mathrm{F}_{12} \mathrm{~A}_{1}=\mathrm{F}_{21} \mathrm{~A}_{2}$, assim pode-se calcular o fator de forma exatamente como: $\mathrm{F}_{12}=\left(\mathrm{r}_{2} / \mathrm{r}_{1}\right)^{2}$. Finalmente, o fator que envolve obstrução fica: $F_{11}=1-F_{12}$.

Na Figura 10 são apresentados os resultados obtidos com a metodologia proposta para o fator de forma $F_{11}$, cada ponto na figura corresponde a uma simulação. Verifica-se que para as duas malhas os resultados numéricos são próximos e concordam bem com o analítico. Observase também que na medida em que o raio externo aumenta, para o raio interno constante, o fator de forma chega a ser praticamente unitário. 


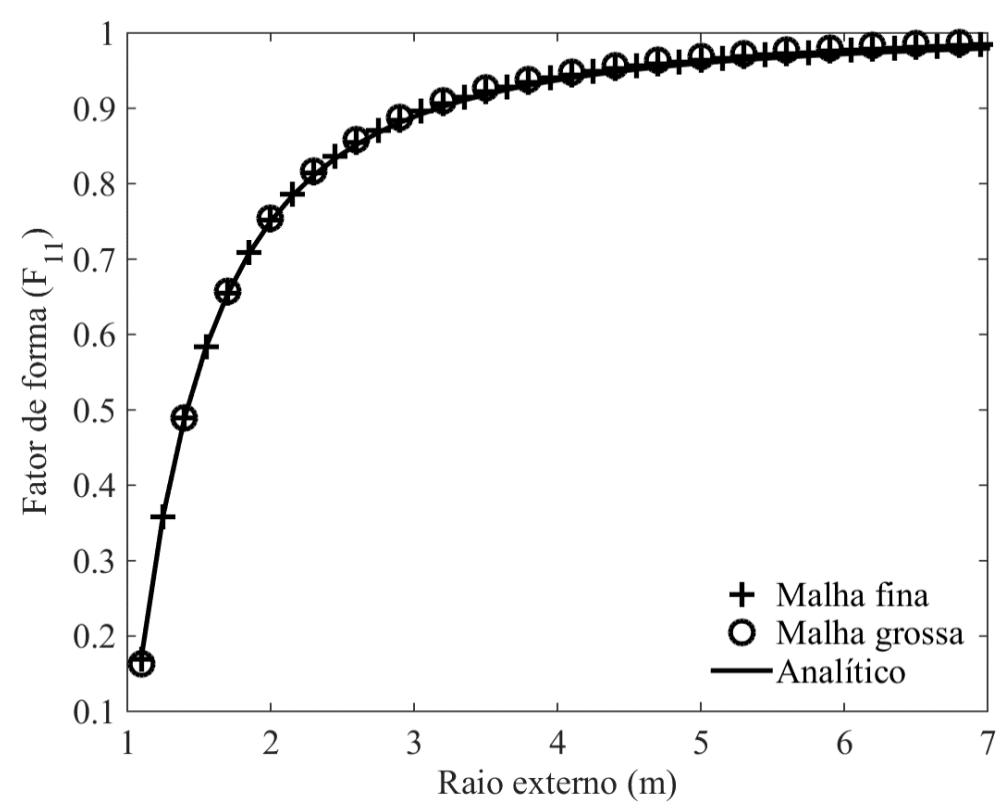

Figura 10. Fator de forma $\mathrm{F}_{11}$.

Na Figura 11 são apresentados os contornos dos elementos triangulares nas superfícies das esferas, colocando em evidência alguns detalhes sobre o cálculo de obstrução. 0 elemento emissor é marcado pela cor preta, os elementos em azul são os obstrutores, os elementos em vermelho são os obstruídos e os elementos em verde são os receptores de radiação. Nenhum elemento marcado de vermelho receberá radiação do elemento emissor devido obstrução dos elementos azuis. Todos elementos obstrutores pertencem a esfera interna. Também se percebe que nem todos os elementos da esfera interna são obstrutores, por isso somente parte da esfera interna aparece na figura.

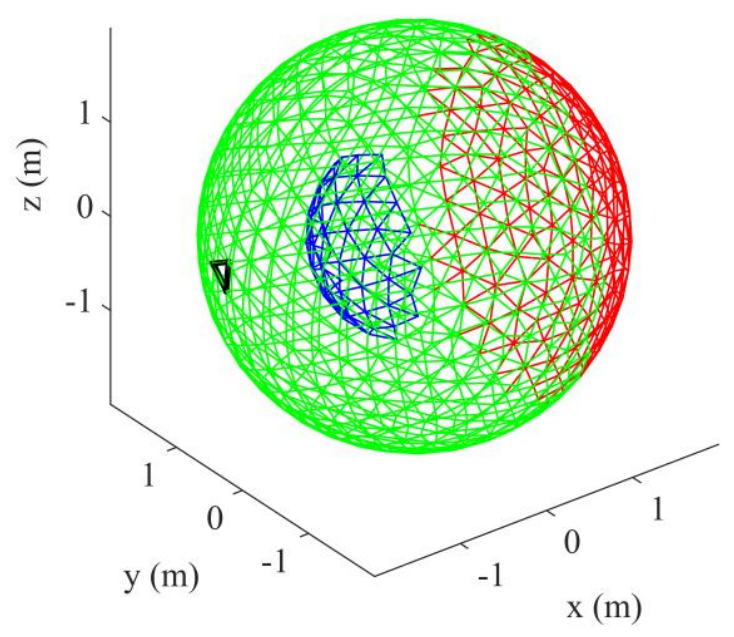

Figura 11. Detalhes da obstrução: Elemento emissor em preto, elementos obstrutores em azul, elementos obstruídos em vermelho e elementos receptores em verde. 


\subsection{Caso de teste 2: Radiador.}

O segundo caso de teste envolve a determinação do fator de forma entre duas aletas de um radiador. Basicamente, o radiador é constituído por aletas interligadas por um tubo, conforme Figura 12. 0 modo como a obstrução de radiação devido o tubo interno ocorre é exemplificado na figura.

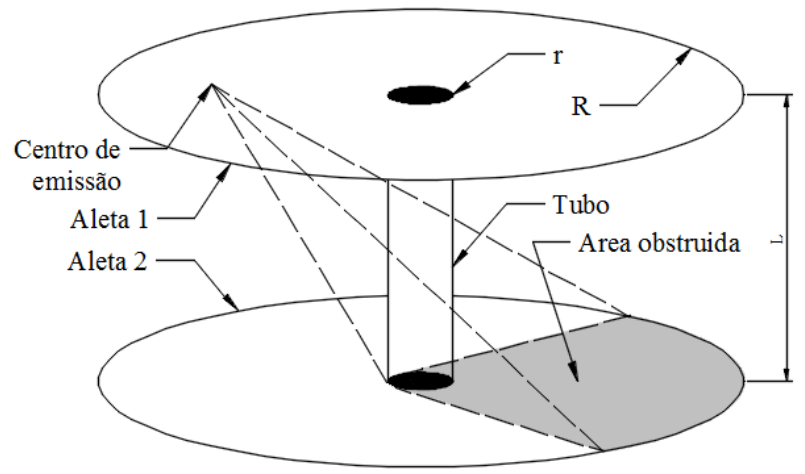

Figura 12. Representação do radiador.

A geometria para os cálculos considera o raio externo $R$ de $6 \mathrm{~cm}$, o raio interno $r$ de $1 \mathrm{~cm}$ e a distância entre os discos $L$ variando entre 1 e $10 \mathrm{~cm}$. Duas malhas de foram geradas, uma malha fina com elementos de $0,3 \mathrm{~cm}$ de tamanho e uma malha grossa com elementos de $0,9 \mathrm{~cm}$ de tamanho. A malha fina é apresentada na Figura 13.

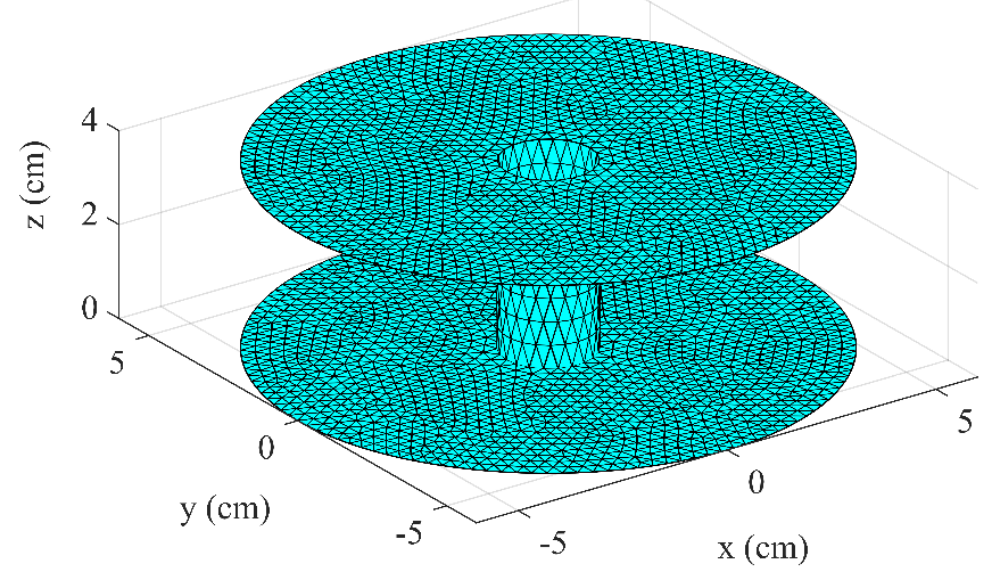

Figura 13. Malha fina do radiador.

Os resultados do cálculo do fator de forma entre as aletas são apresentados na Figura 14 e comparados com os resultados analíticos apresentados em Jithesh, Anil Lal e Rajkumar (2007). Cada ponto na Figura 14 corresponde a uma simulação. Verifica-se que os valores preditos são próximos do resultado analítico e que o fator de forma diminui com o aumento da distância entre 
as aletas. Os resultados obtidos com a malha mais fina são ligeiramente melhores. Constata-se neste caso que a metodologia de cálculo do fator de formar gera resultados precisos mesmo para malha grossa.

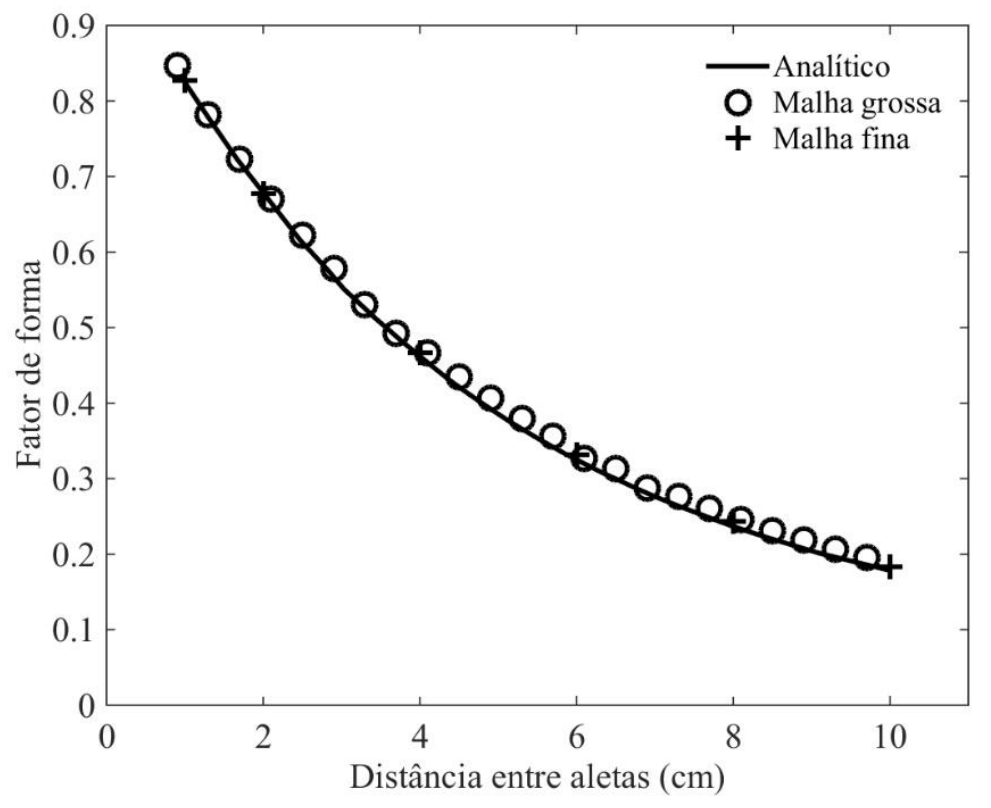

Figura 14. Fator de forma em função da distância entre as aletas.

Alguns detalhes sobre obstrução de radiação são apresentados na Figura 15, na qual, um elemento emissor é marcado pela cor preta, os elementos obstruídos pelo tubo interno são marcados pela cor vermelha e os não obstruídos são marcados pela cor azul. Para cada elemento emissor são verificados os elementos receptores e os obstruídos, assim, o que é apresentado na Figura 15 representa somente a etapa do cálculo associada a emissão de um único elemento.

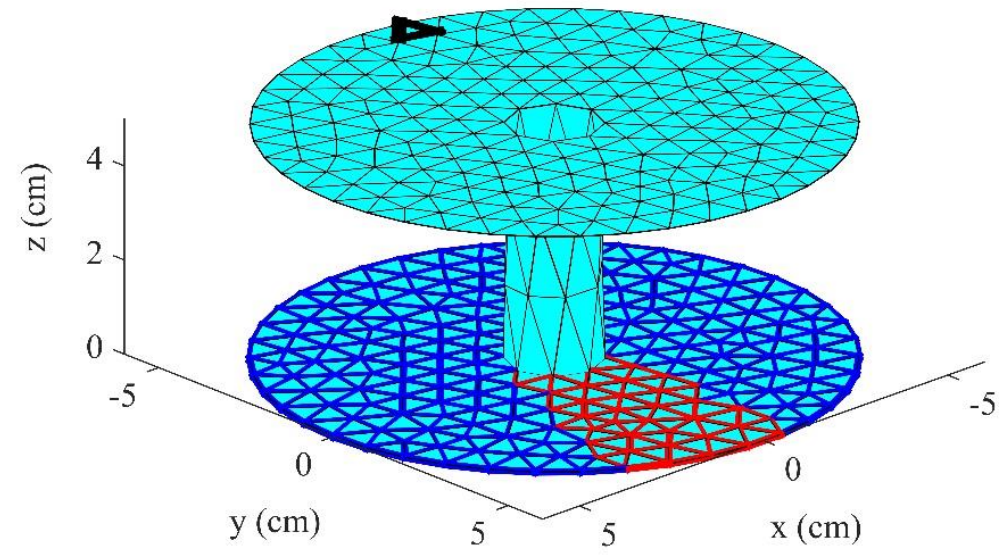

Figura 15. Destaque de um elemento emissor (preto), elementos receptores (azul) e elementos obstruídos (vermelho). 


\section{CONCLUSÃO}

Neste trabalho uma metodologia de cálculo do fator de forma considerando efeitos de obstrução para geometrias tridimensionais foi apresentada e testada.

A metodologia foi baseada em elementos de superfície triangulares, o que torna o método aplicável a malhas computacionais que usam elementos tetraédricos.

Um código numérico foi escrito na linguagem do software Matlab para testar a metodologia de cálculo do fator de forma e verificada boa concordância entre os valores simulados e os resultados analíticos disponíveis na literatura, mesmo para malhas grosseiras.

\section{REFERÊNCIAS BIBLIOGRÁFICAS}

ARAÚJO, F. C.; DA CUNHA, F. A. Metodologia numérica de cálculo do fator de forma considerando os efeitos de obstrução. Revista Interdisciplinar de Pesquisa em Engenharia, v. 02, n. 12, p. 24-43, 2016.

http://periodicos.unb.br/index.php/ripe/article/view/23406.

HOWELL, J. R.; MENGÜÇ, M. P. Radiative transfer configuration factor catalog: A listing of relations for common geometries. Journal of Quantitative Spectroscopy and Radiative Transfer, v. 112, n. 5, p. 910-912. 2011.

http://dx.doi.org/10.1016/i.jqsrt.2010.10.002

JITHESH, P. K.; LAL, S. Anil and RAJKUMAR, M. R. Determination of Radiation View Factors Considering Shadow Effect. Proceedings of 5th International Symposium on Radiative Transfer, p. 2-4. Bodrum, Turkey. 2007.

\section{http://old.ichmt.org/rad-07/Abstracts\%20of\%20posters/P-011.PDF}

JOY, K. Determination of radiative view factor without considering the shadowing effect. Int J Adv Engg Tech. Vol. V/Issue II/April-June, v. 91, p. 95, 2014.

http://www.technicaljournalsonline.com/ijeat/VOL\%20V/IJAET\%20VOL\%20V\%20ISSUE\%2 0II\%20APRIL\%20JUNE\%202014/Article\%2026\%20V\%20II\%202014.pdf

MIYANAGA, Toshiyuki and NAKANO, Yukio. Radiation Heat Transfer in Three-Dimensional Closed Space Including Diffuse and Specular Surfaces. Heat Transfer-Asian Research, 32 (2), 2003.

https://www.jstage.jst.go.jp/article/jsmeb1993/43/3/43 3 449/ article 
YANG, Lei; CHEN, Wenzhen; LUO, Lei and ZHAO, Xinwen. Calculation of Radiation Heat Transfer View Factors Among Fuel Rod Bundles Based on CFD Method. Annals of Nuclear Energy, v. 71, p. 462-466, 2014.

http://dx.doi.org/10.1016/j.anucene.2014.04.024 